\title{
NÚMERO DE POSTOS PLUVIOMÉTRICOS NECESSÁRIOS PARA A ESTIMATIVA DA PRECIPITAÇÃO MENSAL NO ESTADO DO ESPÍRITO SANTO, BRASIL
}

\author{
SAMUEL DE ASSIS SILVA ${ }^{1}$, JULIÃO SOARES DE SOUZA LIMA ${ }^{2}$ \\ ${ }^{1}$ Universidade Federal de Viçosa, Departamento de Engenharia Agrícola (DEA/UFV), Viçosa, MG, Brasil \\ ${ }^{2}$ Universidade Federal do Espírito Santo, Departamento de Engenharia Rural (ERU/UFES), Alegre, ES, \\ Brasil
}

samuel-assis@hotmail.com, limajss@yahoo.com.br

Recebido Maio de 2010 - Aceito Março de 2011

\begin{abstract}
RESUMO
Os objetivos propostos nesse trabalho foram, determinar a distribuição espacial da precipitação pluvial média mensal no Estado, utilizando a altitude dos postos pluviométricos como co-variável e, determinar o número de postos necessários para mapear a precipitação, considerando a dependência espacial. Os dados para realização desse estudo abrangem séries variando de 33 a 72 anos, totalizando 55 postos pluviométricos. Os dados foram submetidos a uma análise descritiva e geoestatística. A co-krigagem usando a altitude como co-variável apresentou considerável acurácia na estimativa da precipitação. A utilização do alcance do variograma cruzado reduziu o número necessário para 37 postos pluviométricos no Estado, para estudar a precipitação média mensal com dependência espacial. Palavras-Chave: Krigagem, Variograma Cruzado, Número de Amostras.
\end{abstract}

\begin{abstract}
NUMBER OF PLUVIOMETRIC GAUGES REQUIRED FOR MONTHLY RAINFALL ESTIMATE OVER THE ESPIRITO SANTO STATE, BRAZIL:

The objectives proposed in this study were to determine the spatial distribution of monthly average rainfall in the state, using the altitude of the rain gauge stations as co-variable and determining the number of points necessary to map the precipitation, considering the spatial dependence. The used data in this study include precipitation series ranging from 33 to 72 years, totaling 55 rain gauge stations. The data series were subjected to descriptive analysis and geostatistics. The co-kriging using the altitude as the covariateble showed considerable accuracy in the estimation of precipitation. The Kriging cross variogram showed that 37 rain gauges are enough to characterize the monthly precipitation with spatial dependence over the State.
\end{abstract}

Keywords: Kriging, Cross Variograms, Number of Samples.

\section{INTRODUÇÃO}

Uma das grandes preocupações da comunidade científica, na última década, diz respeito às alterações climáticas e suas conseqüências para a humanidade (Melo Junior et al., 2006). Conhecer dados de precipitação pluvial é relevante em diversos contextos, tais como: produção agrícola, manejo dos recursos hídricos, avaliação de riscos ambientais, erosão hídrica, etc. A obtenção da correta estimativa da distribuição espacial para a precipitação é primordial no planejamento agrícola, no que diz respeito à instalação de culturas agrícolas anuais. Além da influência na agricultura, períodos de estiagem muito longos afetam o nível de água dos mananciais e dos reservatórios das usinas hidrelétricas, trazendo problemas para o abastecimento urbano e na própria geração de energia elétrica. A quantificação das chuvas com intensidade superior ao suporte do ambiente é importante no planejamento agrícola e ambiental, e para o correto dimensionamento das obras, tanto na construção civil, quanto na conservação do solo (Vieira e Carvalho, 2001; Carvalho e Assad, 2005).

Dentre os métodos de interpolação univariado e multivariados estão os geoestatísticos, os quais devido ao fato de usar a correlação espacial entre observações vizinhas para predizer valores em locais não amostrados, têm sido os mais 
utilizados (Carvalho e Assad, 2005, Kerry e Oliver, 2008). Krigagem simples ou ordinária são os métodos geoestatísticos univariados, que têm sido usado por muitos autores no estudo da distribuição espacial da precipitação pluvial (Tabios e Salas, 1985; Phillips et al., 1992). A extensão multivariada de krigagem, conhecida como co-krigagem, é utilizada quando existe dependência espacial para cada variável em estudo e também entre as variáveis, sendo, portanto, possível utilizar esta técnica na estimativa de valores não amostrados. Esta estimativa pode ser mais precisa do que a krigagem de uma variável simples, quando o variograma cruzado mostrar dependência entre as duas variáveis (Vieira, 2000).

O simples fato de que, por meio da krigagem ou da co-krigagem pode-se conhecer também o erro da estimativa, diferencia-os de qualquer outro método. Esta é uma propriedade interessante, pois, além de permitir a estimativa de valores sem tendência para os locais onde estes não foram medidos, ainda se pode conhecer a confiança associada a estas estimativas, as quais podem ser chamadas de ótimas (Vieira, 2000).

No entanto, não é necessário somente um bom interpolador para obtenção de estimativas mais precisas, mas é fundamental que o sistema de amostragem seja eficiente, o que pode melhorar as predições, além de reduzir custos de implantação, manutenção e monitoramento de postos de coletas de dados. Segundo Godwin e Miller (2008), na maioria das vezes, a população de interesse é demasiadamente grande, tornando-se impossível um levantamento de dados de todos os seus elementos. Nessa situação, deve-se delimitar as observações a uma amostra da população, a qual deve, fielmente, melhor reproduzir suas características.

A eficácia das estratégias de amostragem pode ser aumentada com a incorporação de um modelo de variabilidade espacial, podendo aplicar este conhecimento na escolha da área experimental, locação das unidades experimentais, coleta de dados e interpretação de resultados (Leão et al., 2007).

Conforme o problema exposto e diante da falta de informações para o Estado do Espírito Santo, o trabalho foi desenvolvido procurando alcançar os seguintes objetivos: determinar a distribuição espacial da precipitação pluvial média mensal no Estado, utilizando a altitude dos postos pluviométricos como co-variável, e; determinar o número de postos necessários para mapear a precipitação, considerando a dependência espacial.

\section{DADOS E METODOLOGIA}

O Estado do Espírito Santo situa-se na região Sudeste do país e destaca-se por seu clima bem peculiar, devido à proximidade entre litoral e serra. Ao longo da costa Atlântica encontra-se uma faixa de planície que representa $40 \%$ da área total do Estado e, à medida que se desloca em direção ao interior, o planalto dá origem a uma região serrana, com altitudes superiores a 1.000 metros. O clima é tropical úmido, com temperaturas médias anuais de $23^{\circ} \mathrm{C}$ e volume de precipitação média superior a $2.200 \mathrm{~mm}$ por ano, especialmente concentrada no verão.

Para realização desse estudo foram utilizadas informações obtidos a partir dos postos pluviométricas da Agência Nacional de Águas (ANA) distribuidas por todo o território do Estado, com séries históricas entre 33 a 72 anos de observações de precipitação pluvial de 24 horas expressas em altura de lâmina d'água (mm), totalizando 55 postos pluviométricos com dados consistentes.

As análises foram realizadas, levando-se em conta os valores de precipitação acumulada de cada mês da série, medidos em cada posto pluviométrico. Os totais dos anos foram dividos pelo número de anos de informação para cada posto e, em seguida, pelo número de meses do ano (12), obtendo-se os valores da precipitação pluvial média mensal.

Os dados foram submetidos a uma análise descritiva e a hipótese de normalidade foi testada pelo teste de Shapiro-Wilk's $(p \leq 0,05)$. A dependência espacial entre valores de precipitação pluvial foi calculada pela semivariância, dentro da qual se buscou avaliar o atendimento da hipótese de estacionaridade assumida por meio de ajuste do variograma estimado pela seguinte equação:

$$
\gamma(h)=\frac{1}{2 N(h)} \sum_{i=1}^{N(h)}[Z(x i)-Z(x i+h)]^{2}
$$

em que: $N(h)$ é o número de pares de valores $\left[Z\left(x_{i}\right), Z\left(x_{i}+\right.\right.$ h)] separados por um vetor $h$, e $x_{i}$ é uma posição espacial da variável $Z$, que corresponde à precipitação média mensal. O gráfico de $\gamma(\mathrm{h})$ versus os valores correspondentes de $\mathrm{h}$, chamado variograma, é uma função do vetor $h$, e portanto depende da magnitude e direção de h. Modelos teóricos como o esférico, o exponencial e o gaussiano foram ajustados aos dados experimentais, definindo os parâmetros conhecidos como: efeito pepita $\left(\mathrm{C}_{0}\right)$, correspondendo ao valor da interseção no eixo das semivariâncias; patamar $\left(\mathrm{C}_{0}+\mathrm{C}\right)$, aproximadamente igual ao valor da variância dos dados; e alcance (a), que representa a distância a partir da qual os dados são independentes.

$\mathrm{O}$ ajuste dos modelos foi com base na menor soma de quadrados dos resíduos (SQR), maior coeficiente de determinação $\left(R^{2}\right)$ e o maior coeficiente de correlação entre os valores observados e os estimados pelo método da validação cruzada. Sendo que, a validação cruzada é um método que após escolha do variograma cada valor conhecido é excluído e estimado com os restantes. Para facilitar a comparação, os variogramas utilizados neste trabalho foram escalonados pela variância dos dados como sugerido por Vieira et al. (1991). As análises geoestatísticas e dos métodos de interpolação foram realizadas pelo programa $\mathrm{GS}^{+}$(Robertson, 2000), e os mapas de precipitação construídos no software Surfer. 
Para estimar valores de precipiatação em locais não amostrados e confeccionar os mapas temáticos, utilizou-se a extensão multivariada da krigagem ordinária, a co-krigagem (CoKRIG) adotando a altitude como co-variável.

A krigagem, é um método geoestatístico, que se utiliza de um estimador linear não-viciado com mínima variância e leva em consideração a estrutura de variabilidade encontrada para o atributo e é definido pela seguinte equação (Vieira, 2000):

$$
Z *(x i, x j+h)=\sum_{i=1}^{n} \lambda i Z(x i, x j+h),
$$

sujeito a $\sum_{i=1}^{n} \lambda i=1$,

em que: $Z^{*}\left(x_{i}, x_{i}+h\right)$ é o estimador para um ponto $\left(x_{i}, x_{i}+h\right)$ da região e $\lambda_{i}$ são os pesos usados na estimativa para cada $Z\left(\mathrm{x}_{\mathrm{i}}\right)$.

Krigagem ordinária é o método geoestatístico univariado, que tem sido usado no estudo da distribuição espacial de precipitação pluvial para interpolação de pontos não amostrados (Carvalho e Assad, 2005). A extensão multivariada de krigagem, conhecida como co-krigagem, é utilizada quando existe dependência espacial para cada variável em estudo e também entre as variáveis, sendo portanto possível utilizar esta técnica na estimativa de valores não amostrados (Carvalho et al., 2002; Silva et al., 2010). Esta estimativa pode ser mais precisa do que a krigagem de uma variável simples, quando o variograma cruzado mostrar dependência entre as duas variáveis (Vieira, 2000).

Na co-krigagem (Vieira, 2000), para estimar valores, $\mathrm{Z}_{2}{ }^{*}$, para qualquer local, $\mathrm{X}_{0}$, o valor estimado deve ser uma combinação linear de ambos $Z_{1}$ e $Z_{2}$, ou seja:

$$
z_{2} *\left(x_{0}\right)=\sum_{i=1}^{N 1} \lambda_{1 i} z_{1}\left(x_{1 i}\right)+\sum_{j=1}^{N 2} \lambda_{2 j} z_{2}\left(x_{2 j}\right)
$$

em que: $N_{1}$ e $N_{2}$ são os números de vizinhos de $\mathrm{Z}_{1}$ e $\mathrm{Z}_{2}$, respectivamente, $\lambda_{1} \mathrm{e} \lambda_{2}$ são os pesos associados a cada valor de $\mathrm{Z}_{1}$ e $\mathrm{Z}_{2}$. Tomando $\mathrm{z}_{1}\left(\mathrm{x}_{1 \mathrm{i}}\right)$ e $\mathrm{z}_{2}\left(\mathrm{x}_{2 \mathrm{j}}\right)$ como sendo uma realização das funções aleatórias $Z_{1}\left(X_{1 i}\right)$ e $Z_{2}\left(X_{2 j}\right)$, respectivamente, e assumindo estacionaridade de ordem 2 , o estimador pode ser reescrito como:

$$
Z_{2} *\left(x_{0}\right)=\sum_{i=1}^{N 1} \lambda_{1 i} Z_{1}\left(X_{1 i}\right)+\sum_{j=1}^{N 2} \lambda_{2 j} Z_{2}\left(X_{2 j}\right)
$$

expressando que a estimativa da variável $Z_{2}{ }^{*}$ deverá ser uma combinação linear de ambos $Z_{1}$ e $Z_{2}$, com os pesos $\lambda_{\text {: e }} \lambda_{Z}$ distribuídos de acordo com a dependência espacial de cada uma das variáveis entre si e a correlação cruzada entre elas.

O desempenho dos interpoladores foi obtido e comparado com base nos valores de erro padrão da estimativa e do coeficiente de correlação da validação cruzada, segundo o modelo de variograma teórico ajustado.

Para efeito de comparação com a metodologia espacial utilizada, determinou-se, segundo a Estatística clássica, o número de amostras (postos pluviométricos) representativa para estimar a precipitação pluvial média mensal no Estado. Essa determinação foi realizada segundo proposto por Cochran (1965). Apesar do cálculo de amostras aleatórias simples não considerar as alterações de relevo e as irregularidades naturais dos terrenos, esse método é útil para indicar, estatisticamente, se a utilização da co-krigagem apresenta eficiência na determinação do número de postos pluviométricos.

$$
\mathrm{n}=\frac{\mathrm{t}^{2} \alpha^{*} \mathrm{~S}^{2}}{\mathrm{~d}^{2}}
$$

em que: $n$ é o número de amostras necessário para estimar a média, dentro de uma tolerância de $5 \%, 10 \%, 15 \%, 20 \%, 25 \%$, e $30 \%$; $t_{\alpha}$ é o valor do teste t de Student ao nível de $95 \%$ de confiança e $\mathrm{S}^{2}$ a variância amostral.

Para definir o número de subamostras necessárias para avaliar a precipitação pluvial média mensal, considerou-se o alcance obtido por meio do variograma cruzado das variáveis precipitação versus altitude, na co-krigagem. Para garantir a dependência espacial, assumiu-se que os pontos de amostragens (postos pluviométricos) deveriam ser aqueles a uma distância equivalente à metade do alcance determinado, conforme Carvalho e Assad (2005) e Souza et al. (2006).

Tabela 1- Estatística descritiva da precipitação média e altitude em 55 postos pluviométricos no Estado do Espírito Santo.

\begin{tabular}{cccccccccccc} 
Variáveis & Média & Mediana & Mín & Máx & S & CV(\%) & Cs & Ck & p-valor Correlação \\
\hline Altitude & 260,81 & 127,00 & 0,00 & 940,00 & 280,57 & 107,58 & 1,07 & $-0,13$ & 0,00 & 0,30 \\
Precipitação & 108,64 & 79,0 & 0,00 & 962,2 & 99,4 & 91,45 & 1,60 & 3,40 & 0,01 &
\end{tabular}

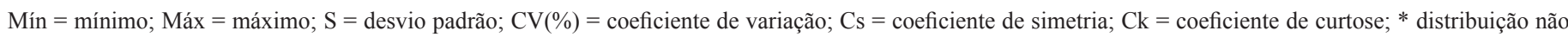
normal pelo teste Shapiro-Wilk's $(\mathrm{p} \leq 0,05)$. 

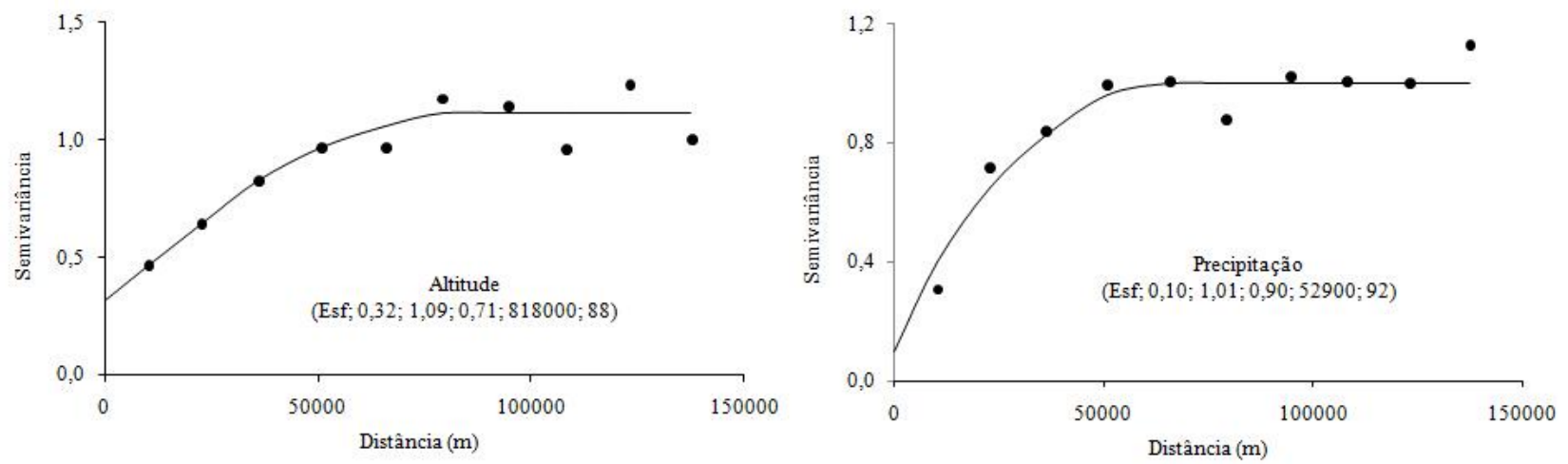

Figura 1- Modelos e parâmetros $(\mathrm{C} 0 ; \mathrm{C} 0+\mathrm{C}$; IDE; a; R2) dos variogramas escalonados da altitude e precipitação média para o Estado do Espírito Santo.

\section{RESULTADOS}

As principais estatísticas do Estado do Espírito Santo no período estão na Tabela 1. A média e a mediana apresentaram-se distantes para as duas variáveis, indicando uma distribuição assimétrica dos dados.

Os dados de precipitação apresentaram distribuição leptocúrtica, enquanto que os dados de altitude distribuição platicúrtica. Estas estatísticas indicaram a não normalidade dos dados, o que foi confirmado pelo teste Shapiro-Wilk's $(\mathrm{p} \leq 0,05)$. De acordo com Cressie (1991) a normalidade dos dados não é exigência da geoestatística, e sim conveniente apenas que a distribuição não apresente extremidades muito alongadas, o que poderia comprometer as análises.

Os coeficientes de variação das duas variáveis são considerados altos (Tabela 1). Essa variação evidencia o efeito das baixas precipitações ocorridas na região norte e das maiores na região serrana do Estado e da alternância na altitude. Verifica-se que a correlação linear entre a altitude e a precipitação, apesar de numericamente baixa e positiva, é altamente significativa $(\mathrm{p} \leq 0,05)$, conforme discutido por Carvalho e Assad (2005),

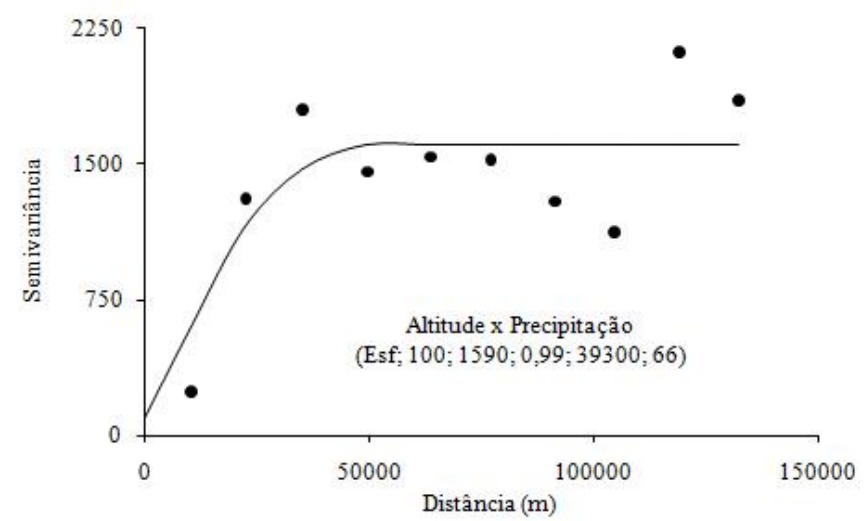

Figura 2- Modelos e parâmetros (C0; $\mathrm{C} 0+\mathrm{C}$; IDE; a; $\mathrm{R} 2)$ do variograma cruzado da altitude e precipitação para o Estado do Espírito Santo. indicando que se pode utilizar a altitude como co-variável na determinação da distribuição espacial da precipitação.

Na Figura 1, os variogramas na análise geoestatística indicam que as variáveis apresentam dependência espacial, ajustando-se as semivariâncias dessas variáveis o modelo esférico, com alcance de $81.800 \mathrm{~m}$ e $52.900 \mathrm{~m}$ para a altitude e a precipitação média, respectivamente. Em um estudo semelhante realizado por Carvalho e Assad (2005), no Estado de São Paulo, o modelo ajustado para a altitude foi o exponencial. Nesses casos, os pontos localizados numa área com raio menor ou igual ao alcance apresentam precipitações similares e estão espacialmente dependentes entre si, podendo estimar com maior precisão valores não medidos nessa área.

Em função da correlação significativa da precipitação média mensal com a altitude, construiu-se o variograma cruzado entre essas variáveis, de forma a estimar valores de precipitação média mensal em toda a área do Estado (Figura 2), obtendo-se um alcance de $39.300 \mathrm{~m}$, efeito pepita $\left(\mathrm{C}_{0}\right)$ de 100 e patamar $\left(\mathrm{C}_{0}\right.$ + C) de 1.590. Apesar do menor alcance encontrado, verificou-se um elevado índice de dependência espacial (IDE), obtido pela relação $\mathrm{C} /\left(\mathrm{C}_{0}+\mathrm{C}\right)$, de 0,99 , classificada como forte dependência.

$\mathrm{Na}$ Figura 3, verificam-se os mapas temáticos da distribuição da altitude por krigagem e da precipitação por co-Krigagem. A maior variabilidade espacial da altitude está na região serrana do Estado, de acordo com o comportamento das isolinhas (mais próximas). Observa-se que a zona de maior precipitação não coincide, na totalidade, com a região de maiores altitudes, o que vem a confirmar a correlação de 0,30 entre essas variáveis, apesar de significativa.

Analisando os mapas temáticos de precipitação construídas por co-krigagem, observa-se que os menores índices ocorrem na região norte, oeste e parte do sul; e com os maiores índices, em parte, no litoral sul e na região serrana. Parte considerável do território do Estado apresenta precipitação média mensal variando entre 95 a $115 \mathrm{~mm}$. A observância dessa variação na distribuição da precipitação é informação essencial para o planejamento 

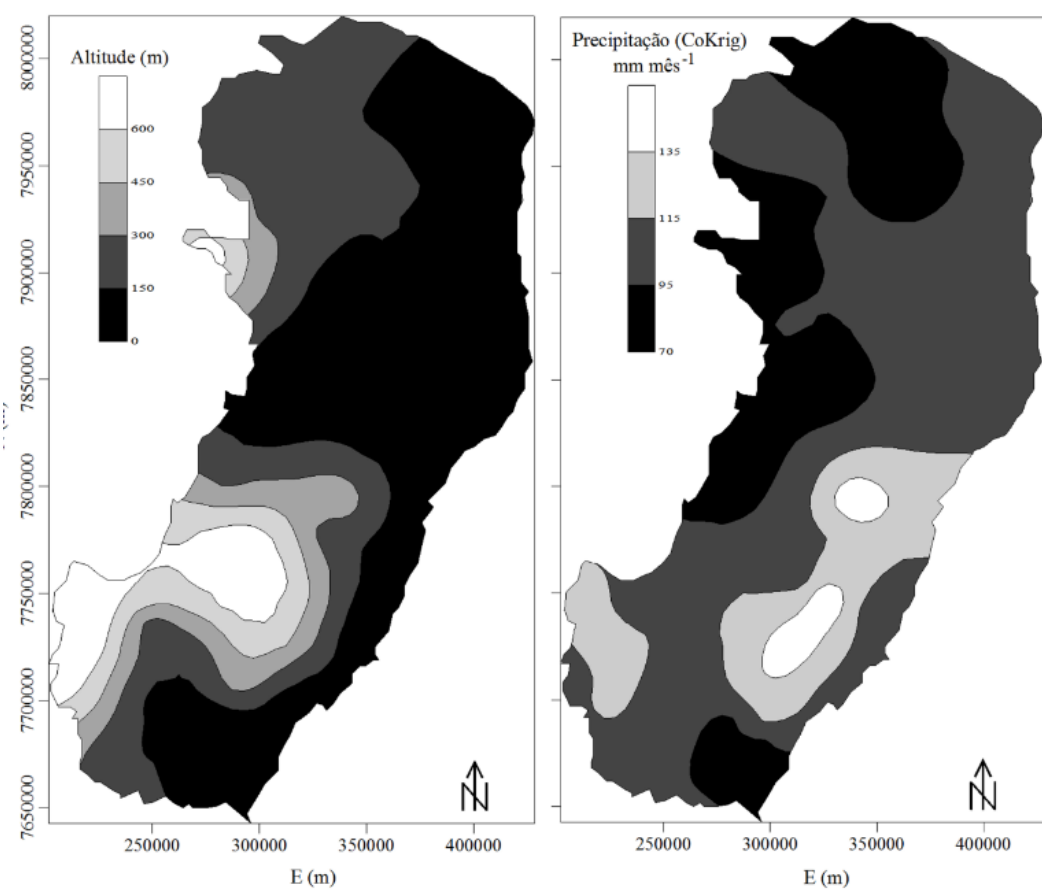

Figura 3- Mapas temáticos para as variáveis altitude e precipitação (CoKRIG) para o Estado do Espírito Santo.

agrícola, como no estudo de zoneamento, previsão de safras, caracterização climatológica, etc. Para o Estado de São Paulo, Carvalho e Assad (2005) definiram que a maior precipitação ocorrida em região montanhosa deve-se aos ventos úmidos vindo do litoral, que se deparam com a cadeia de montanhas servindo de barreiras naturais obrigando o ar a subir, que se arrefece e se satura de vapor d'água, com a consequente formação de nuvens originando a precipitação de relevo, ou orográfica.

Os números de postos pluviométricos necessários para registro da precipitação pluvial média mensal no Estado estão apresentados na Tabela 2. Considerando a Equação 5 e adotando o desvio de $5 \%$ em torno da média, tem-se 1.286 postos. Porém, para garantir a dependência espacial, assumiu-se que os postos devem ser localizados a uma distância equivalente à metade do alcance obtido na co-krigagem, ou seja, a cada $20.000 \mathrm{~m}$ (uma área circular de $1256 \mathrm{~km}^{2}$ ). Sabendo-se que a área do Estado é de, aproximadamente, $46.078 \mathrm{~km}^{2}$, então seriam necessários no minimo 37 postos distribuídos aleatoriamente no Estado. Segundo Carvalho e Assad (2005), normalmente, a Estatística clássica requer maior número de amostras do que a Goestatística, para estimar uma determinada variável com precisão.
Utilizando o alcance para estimar a precipitação média mensal com dependência espacial o número de postos diminuiu de 55 para 37. Cabe ressaltar que, quanto maior o número de postos tem-se maior precisão na estimativa de valores para locais não amostrados.

A Figura 4 apresenta a distribuição dos 55 postos pluviométricos no Estado em função do alcance de dependência espacial determinado pela co-krigagem, indicando áreas em que as precipitações ali ocorridas são representativas e em outras com baixa precisão. Locais onde ocorre a interseção entre as circunferências mostram boa precisão na estimativa de valores em locais sem a presença de postos. Cabe ressaltar que somente foram utilizados nesse estudo os postos da ANA com mais de 30 anos de observações consecutivas, o que influiu na cobertura total no Estado, apresentando regiões com baixa precisão na estimativa da precipitação.

\section{CONCLUSÕES}

O uso da altitude como co-variável no estudo da precipitação pluvial média mensal, permitiu a obtenção de

Tabela 2- Número mínimo de amostras necessário para estimar os valores de precipitação pluvial, nas diferentes porcentagens em torno da média.

\begin{tabular}{llllllll}
\hline D.M & $\mathbf{5 \%}$ & $\mathbf{1 0 \%}$ & $\mathbf{1 5 \%}$ & $\mathbf{2 0 \%}$ & $\mathbf{2 5 \%}$ & $\mathbf{3 0 \%}$ & $\begin{array}{l}\text { Considerando } \\
\text { o Alcance }\end{array}$ \\
\hline N.P & 1286 & 322 & 143 & 81 & 52 & 36 & 37 \\
\hline
\end{tabular}

D.M: desvio em torno da média e N.P: número de postos pluviométricos 


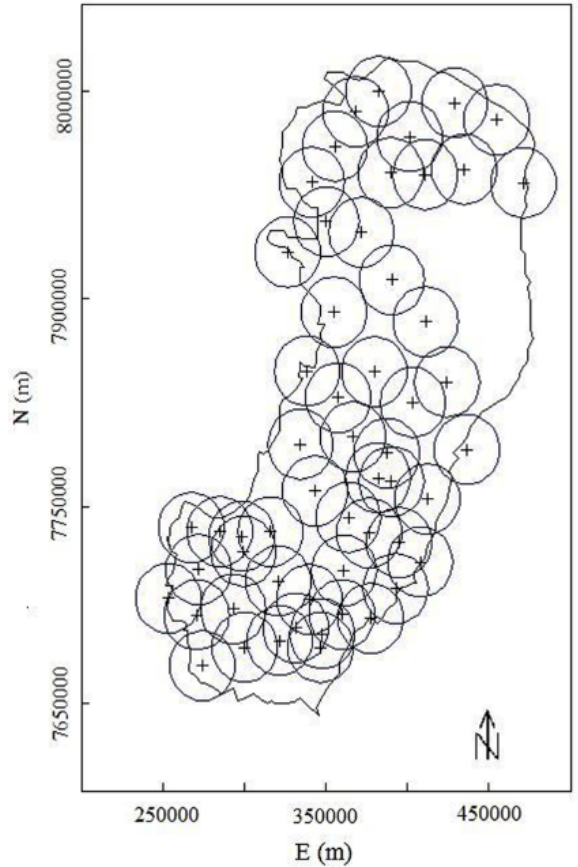

Figura 4 - Distribuição espacial dos postos pluviométricos estudados sob a influência do alcance da dependência espacial no registro da precipitação pluvial no ES.

resultados satisfatórios na estimativa de valores em locais não amostrados, sendo capaz de determinar e expressar a continuidade espacial do fenômeno.

É possível utilizar métodos geostatísticos multivariados para determinar o número de postos pluviométricos necessários para caracterizar a distribuição espacial da precipitação e, principalmente, através do alcance dos variogramas cruzados, orientar a distribuição destes ao longo dos territórios a serem mapeados.

O estudo apresentado sugere que o levantamento da precipitação não é dependente apenas do número de postos pluviométricos, mas principalmente da distribuição dos mesmos ao longo da área a ser amostrada, sendo o modelo ideal de mapeamento aquele que considera de forma conjunta um número reduzido de postos, distribuídos de maneira adequada.

\section{REFERÊNCIAS BIBLIOGRÁFICAS}

CARVALHO, J. R. P.; ASSAD, E. D. Análise espacial da precipitação pluviométrica no Estado de São Paulo: Comparação de métodos de interpolação. Engenharia Agrícola, v. 25, n. 2, p. 377-384. 2005.

CARVALHO, J. R. P.; SILVEIRA, P. M.; VIEIRA, S. R. Geoestatística na determinação da variabilidade espacial de características químicas do solo sob diferentes preparos. Brasília. Pesquisa Agropecuária Brasileira, v. 37, n. 8, p. 1151-1159, 2002.
COCHRAN, W. G. Téenicas de amostragem. Rio de Janeiro: Fundo de Cultura, 1965. 555p.

CRESSIE, N. Statistics for spatial data. New York: John Wiley, 1991. $900 \mathrm{p}$.

GODWIN, R. J.; MILLER, P. C. H. A review of the technologies for mapping within-field variability. Biosystems Engineering, v. 84, p. 393-407. 2008.

KERRY, R.; OLIVER, M. A. Determining nugget: sill ratios of standardized variograms from aerial photographs to krige sparse soil data. Precision Agriculture, v. 9, p. 33-56, 2008.

LEÃO, A. B.; ANDRADE, A. R. S. de; CHAVES, L. H. G; CHAVES, I. B.; GUERRA, H. O. C. Variabilidade espacial de fósforo em solo do perímetro irrigado Engenheiro Arcoverde, PB. Revista Ciência Agronômica, v. 38, n. 1, p. 1-6, 2007.

MELO JÚNIOR, J. C. F. de; SEDIYAMA, G. C.; FERREIRA, P. A.; LEAL, B. G.; MINUSI, R. B. Distribuição espacial da freqüência de chuvas na região hidrográfica do Atlântico, Leste de Minas Gerais. Revista Brasileira de Engenharia Agrícola e Ambienta, v. 10, n. 2, p.417-425, 2006.

PHILLIPS, D. L.; DOLPH, J.; MARKS, D. A comparison of geostatistical procedures for spatial analysis of precipitations in mountainous terrain. Agriculture and Forest Meteorology, n. 58, p. 119-141, 1992.

ROBERTSON, G. P. GS+: Geostatistics for the environmental sciences - GS+ User's Guide. Plainwell, Gamma Desing Software, 2000. $152 \mathrm{p}$.

SILVA, S. A.; LIMA, J. S. S.; SOUZA, G. S. Estudo da fertilidade de um Latossolo Vermelho-Amarelo húmico sob cultivo de café arábica por meio de geoestatística. Revista Ceres, v. 57, n.4, p. 560-567, 2010.

SOUZA, Z. M; MARQUES JUNIOR, J.; PEREIRA, G. T.; MONTANARY, R. Otimização amostral de atributos de latossolos considerando aspectos solo-relevo. Ciência Rural, v. 36, n. 3, p. 892-836. 2006.

TABIOS, G. Q.; SALAS, J. D. A comparative analysis of techniques for spatial interpolation of precipitation. Journal of the American Water Resources, v. 21, n. 3, p. 365-380, 1985.

VIEIRA, S. R.; CARVALHO, J. R. P. de. Estudo da periodicidade temporal de chuvas em bacia hidrográfica dos Rios Turvo/Grande - uma proposta. Campinas: Embrapa Informática Agropecuária, 2001. 17 p. (Embrapa Informática Agropecuária. Documentos, 10).

VIEIRA, S. R. Geoestatística em estudos de variabilidade espacial do solo. In: NOVAIS, R.F. de; ALVAREZ V.H.; SCHAEFER, C.E.G.R. Tópicos em ciência do solo, Viçosa - MG: Sociedade Brasileira de Ciência do Solo, 2000. v.1, p.1-54.

VIEIRA, S. R.; LOMBARDI NETO, F.; BURROWS, I. Mapeamento das precipitações máximas prováveis para o Estado de São Paulo. Campinas, Revista Brasileira de Ciência do Solo, v. 15, n. 1, p. 93-98, 1991. 